\title{
Images of the month 2: Gastrointestinal bleeding with a large liver and a small spleen: a rare appearance of multiple myeloma
}

Authors: Feng Feng, ${ }^{\mathrm{A}}$ Ya Liu, ${ }^{\mathrm{B}}$ Xuehui $\mathrm{Cao}^{\mathrm{C}}$ and Jianhua Liu ${ }^{\mathrm{D}}$
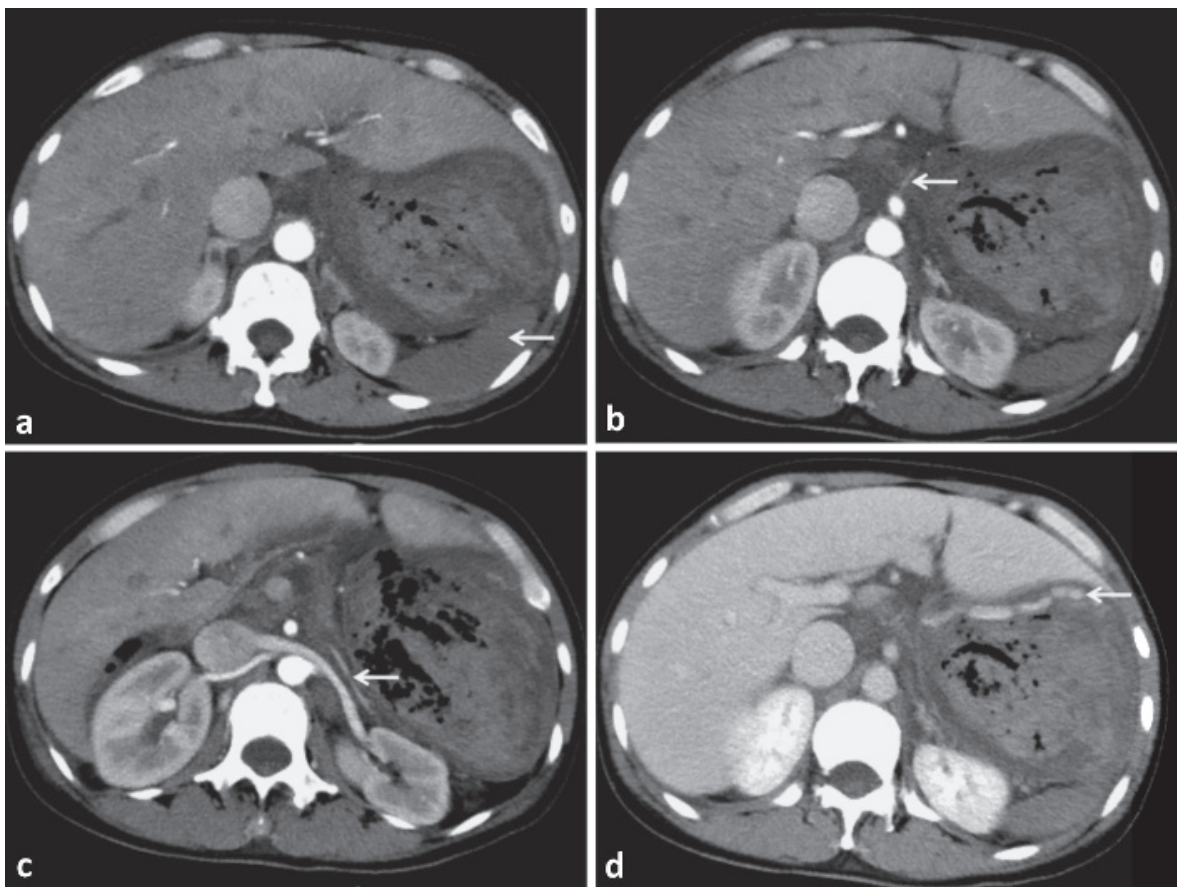

Fig 1. Contrast-enhanced computed tomography prior to emergency surgery. a) The arrow indicates delayed contrast enhancement in the shrunken spleen. b) The arrow shows a fine left gastric artery arising from the celiac trunk. c) The arrow indicates a fine splenic artery above the pancreas. d) The arrow shows a swollen gastric wall and contrast exposure in the stomach.

KEYWORDS: Gastrointestinal bleeding, multiple myeloma

\section{Case presentation}

A 38-year-old woman presented to the emergency department because of sudden-onset massive haematemesis. Contrastenhanced computed tomography indicated gastrointestinal (GI)

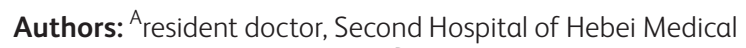
University, Shijiazhuang, China; ${ }^{B}$ associate professor, Second Hospital of Hebei Medical University, Shijiazhuang, China; ${ }^{C}$ resident doctor, Second Hospital of Hebei Medical University, Shijiazhuang, China; D professor, Second Hospital of Hebei Medical University, Shijiazhuang, China bleeding with an enlarged liver and an unexpectedly-shrunken spleen (Fig 1). Laboratory studies revealed anaemia (haemoglobin of $5.9 \mathrm{~g} / \mathrm{dL}$ ), proteinuria (urine protein +++ ), hypercalcaemia $\left(\mathrm{Ca}^{2+}\right.$ of $\left.2.77 \mathrm{mmol} / \mathrm{L}\right)$, and a significantly increased serum $\beta 2$ microglobulin level (4.1 mg/L, normal range $1.3-2.7 \mathrm{mg} / \mathrm{L}$ ). Gastroscopy showed massive blood clots in the stomach and no clear bleeding site could be found.

We performed an emergency laparotomy and confirmed the imaging findings. During surgery, the gastrocolic ligament was transected from the junction between gastroepiploic arteries to the left side of the cardia. The hepatoduodenal ligament was transected from the incision of gastric angle to the right side of the cardia.

Based on the abnormal finding of a large liver and a small spleen, the patient may have had undiscovered systemic diseases, so we asked a haematologist for consultation. Multiple myeloma was suspected and subsequently confirmed by bone marrow biopsy and liver pathological section (Fig 2). At the last follow-up, the 

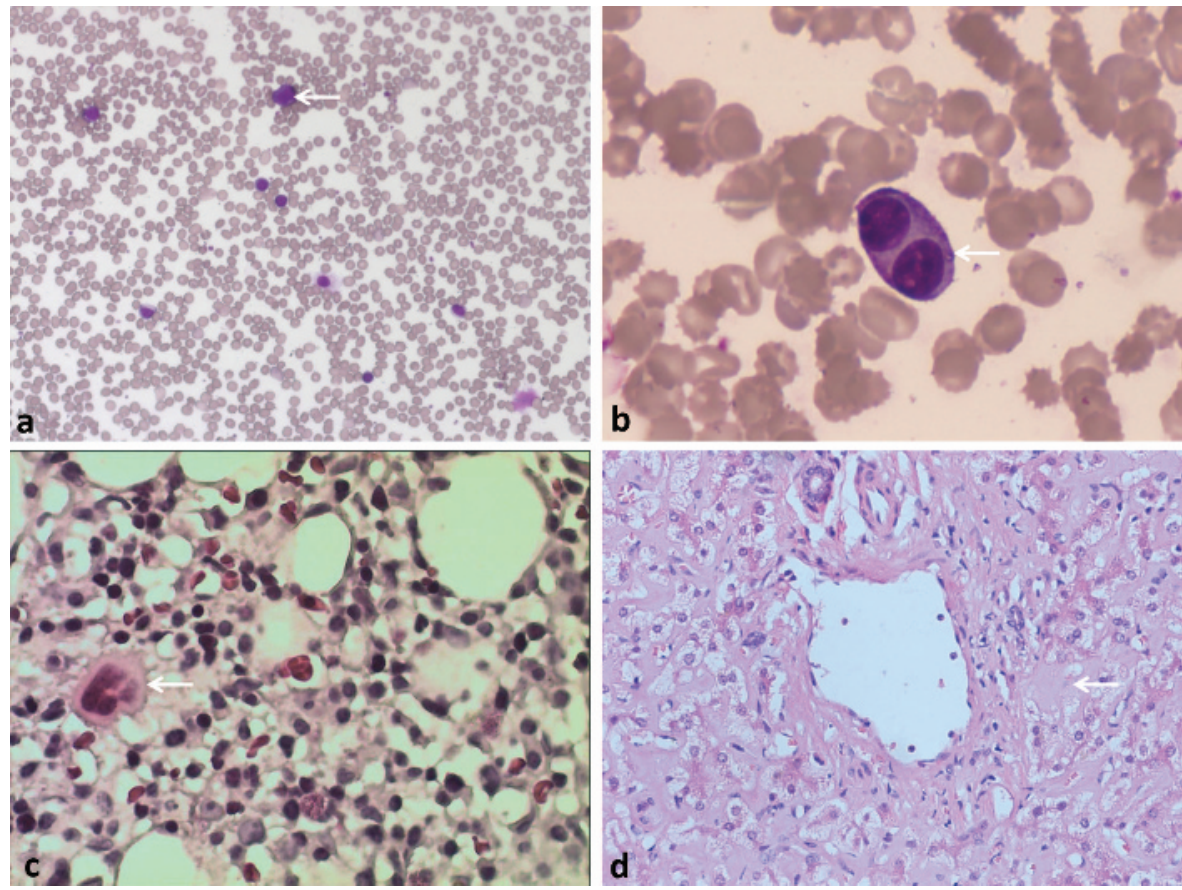

Fig 2. Histological results of the patient's bone marrow and liver. a) Bone marrow smear (haematoxylin and eosin stained, $\times 200$ ) showed obviously active proliferation of bone marrow nucleated cells with granulocytes predominantly in the middle and late stage and obviously increased plasma cells (the arrow) with naive plasma cells, binuclear plasma cells and abnormal nuclear plasma cells (naive plasma cell 8.5\%; mature plasma cell 42.5\%). b) Bone marrow smear (haematoxylin and eosin stained, $\times 400$ ) showed a typical binuclear plasma cell (the arrow) with large cell body, a large amount of cytoplasm, round or oval nucleus, nuclear deviation, and coarse chromatin. c) Bone marrow biopsy showed significantly increased plasma cells (the arrow) and the immunohistochemical examination showed positive results in CD138, CD38, Cyclin D1, and $\kappa$ and negative results in CD56, CD19, CD117, CD20, and $\lambda$. d) Liver pathological section revealed that the liver cells were partially oedematous and partially atrophic with diffused pink-coloured, non-structural substances (the arrow) in the liver sinuses and the immunohistochemical examination showed positive results in CD138, $\kappa$, Ckpan, and Ki-67 (+15\%) and negative results in alpha-fetoprotein, CD10, CD34, CD38, CD68, and $\lambda$. Special staining of the liver showed positive results in reticular fibre staining, periodic acid-Schiff staining, Masson's trichromatic staining, and weakly positive results in Congo red staining.

patient was in good general condition and receiving her second chemotherapy course.

\section{Discussion}

GI bleeding is extremely rare as the initial manifestation of multiple myeloma, and in this patient, enlargement of liver and reduction of spleen also occurred, which made this case extremely rare. ${ }^{1}$ Several possible explanations have been reported with regard to GI bleeding in multiple myeloma patients. First, the deposition of amyloid protein in the GI wall leads to increased capillary fragility. Additionally, myeloma cells may directly infiltrate the GI tract in the form of plasmacytoma and cause mucosal hyperplasia, oedema, erosion and repair dysfunction. ${ }^{2,3}$

Non-haematologists may not have sufficient understanding of multiple myeloma and often miss further examination, resulting in misdiagnosis. For patients suffering GI bleeding combined with an enlarged liver, an unexpectedly shrunken spleen, and other abnormalities in laboratory tests such as proteinuria, hypercalcaemia and high serum $\beta 2$ microglobulin level, multiple myeloma should be suspected and bone marrow examination should be performed to avoid misdiagnoses.

\section{References}

1 Lin M, Zhu J, Shen H, Huang J. Gastrointestinal bleeding as an initial symptom in asymptomatic multiple myeloma: A case report and review of the literature. Oncol Lett 2013;5:218-20.

2 Maskin LP, Díaz MF, Hlavnicka A et al. Gastrointestinal bleeding secondary to multiple gastric plasmacytoma. Am J Clin Oncol 2008:31:100-1.

3 Nakajima K, Sueki Y, Koshiishi M et al. Gastric invasion of multiple myeloma presenting as gastrointestinal bleeding. Int J Hematol 2015:101:1-2.

Address for correspondence: Prof Jianhua Liu, Hepatobiliary Department, Second Hospital of Hebei Medical University, 215 Hepingxi Road, Shijiazhuang 050000, China. Email: dr.ljh@outlook.com 